\title{
The Association of Brain-Derived Neurotrophic Factor and Malondialdehyde Level with Depression In-Patient Post-Stroke Ischemic
}

\author{
Yuliarni Syafrita ${ }^{1}$, Darwin Amir ${ }^{2}$, Restu Susanti ${ }^{3}$, I Fadilah $^{4}$ \\ \{yuliarni@med.unand.ac.id ${ }^{1}$ \} \\ Faculty of Medicine, Universitas Andalas / DR M Djamil Hospital, Department of Neurology, \\ Padang, West Sumatra, Indonesia
}

\begin{abstract}
Post-stroke depression was a serious psychosomatic complication, and this condition can disrupt the stroke healing process. This study aims to find the association of the levels of DNF and Malondialdehyde (MDA) acute phase of stroke and the effect of vascular risk factors with incident of post-stroke depression. An observational study with the case-control design was carried out in 72 ischemic stroke patients at Dr. M Jamil Hospital in Padang. Levels of BDNF and MDA serum in the acute phase of stroke was carried out using the Elisa method. Based on the result of the Hamilton Depression Rating Scale which was conducted 1 month after onset of stroke, a sample of this study was divided into post-stroke groups with depression and without depression. The differences of mean levels of BDNF and MDA were analyzed using t-test and Mann-Whitney and the differences of the basic characteristics of these two groups were analyzed by chi-square. The mean level of BDNF in the depression group was lower than the group without depression, and the difference was statistically significant with $p$-value $=0.009$. The mean level of MDA in the depression group was higher than the non-depressed group, and this difference was also statistically significant with $p$-value $=0.024$. A lower level of BDNF serum and a higher level of MDA in the acute phase of ischemic stroke is associated with the incidence of post-stroke depression. Patient with diabetes mellitus has a higher risk to suffer post-stroke depression.
\end{abstract}

Keywords: BDNF, Ischemic Stroke, MDA.

\section{Introduction}

Post-stroke depression (PSD) is a serious and very disturbing complication in the stroke recovery process. Various studies show some the incidence rate of stroke is $20-50 \%$, which is quite a high number. Post-stroke depression was found to be associated negatively with clinical and functional outcomes in stroke patients. This condition can cause a decrease in quality of life (QoL), disrupt the activities of daily living (ADL), reduce functional independence, reduce social interaction and also reduce a successful rate of rehabilitation. In addition, post-stroke depression also increases health service burdens and it is related to a higher mortality post-stroke rates. It is also related to cognitive disorders, such as attention, learning abilities, and executive functions, and also delayed physical recovery [1],[2].

The pathogenesis of post-stroke depression is very complex, which involved a variety of specific neurobiologies mechanisms, such as neuroanatomic neurons and biochemical factors 
and also neurogenesis which interacts complexly. Several previous studies have shown that a big lesson in the significant areas such as left frontal lobe and basal ganglia or accumulation of asymptomatic cerebral lesion can affect monoamine pathways or mood control pathways that will lead into depression [3].

Brain-Derived Neurotrophic Factor is a part of neurotrophins and is expressed in the central and peripheral nervous systems of adult mammals, especially in the hippocampus and brain cortex. Brain-Derived Neurotrophic Factor is a widespread neurotrophin in the central nervoussystem[4]. Brain-Derived Neurotrophic Factor has several functions, including axonal regulation, dendritic growth, the response in neurotransmitter release, and long-term potentiation (LTP). According to those function, BDNF has a role as the main regulator of synaptic plasticity [5].

Clinical studies have shown that serum BDNF levels and decreased hippocampal volume in the elderly are correlated closely with memory disorders and neuropsychiatric disorders. Further analysis shows a lower BDNF level can cause a decreased of hippocampal volume which will cause spatial memory loss, and also depression [6].

In addition, various neurodegenerative diseases are also triggered by oxidative stress. Under normal condition, there is a balance between pro-oxidants and anti-oxidants, but under certain conditions, such as reduced blood flow (stroke), a disturbance of this balance will happen, and it will increase reactive oxygen species (ROS) and reactive nitrogen species (RNS) production. These ROS and RNS will interact with various biomolecules such as proteins, lipids, carbohydrates, deoxyribonucleic acids (DNA) and ribonucleic acids (RNA), which will produce various toxic substances[7].

It has been proven that free radicals also play a role in the pathophysiology of depression [8]. Recently, malondialdehyde (MDA), which is the main endogenous product of lipid peroxidation, was found elevated in patients who had been diagnosed with major depression [9]. Stefanescu et al., found a higher MDA concentration in patients with recurrent episodes of depression [10]. Increased levels of MDAwas also found in depression patients with another disease, such as chronic heart failure [11].

Various hypotheses that develop about the pathophysiology of post-stroke depression makes this topic needs more research to conduct. Therefore the authors want to conduct research to see the relationship between BDNF and MDA serum level in the acute phase of stroke with post-stroke depression after 1-month of onset.

\section{Materials and Methods}

This study has passed the ethical study issued by the Research Ethics Committee of the Medical Faculty of Andalas University with the Number: 511 / KEP / FK / 2018. Observational study with a case-control design was conducted in 72 post-ischemic stroke patient, which consist of 36 post-stroke patients with depression and 36 patient without depression at DR M Djamil Padang Hospital from January until September 2018. Inclusion criteria of this study were patient with ischemic stroke with onset $<72$ hours, age $<70$ years old, no history of depression before the onset of stroke and the symptoms of depression was persist for $>2$ weeks, not suffering from the other neurodegenerative diseases like Alzheimer and Parkinson. The examination of depression was conducted using Hamilton Depression Rating Scale in 1 month after onset of stroke, based on this examination result; the patient was divided into two groups which are poststroke groups with depression and group without depression. Levels of BDNF and MDA was 
examined using the Elisa method. The differences of mean levels of these two groups were tested by t-test and Mann-Whitney test, and the differences of the basic characteristic of these two groups were tested by the chi-square test.

\section{Results}

Table 1. The basic characteristic of those two groups can be seen below

\begin{tabular}{lllc}
\hline & \multicolumn{2}{l}{ Post Ischemic Stroke } & \multirow{2}{*}{$\mathrm{P}$} \\
\cline { 2 - 3 } & With Depression & Without Depression & \\
\hline Age & $59.67 \pm 9,7$ & $59.64 \pm 11,2$ & $0.85^{\mathrm{a}}$ \\
Sex: Male/Female & $18 / 18$ & $19 / 17$ & $0.814^{\mathrm{a}}$ \\
Education Level: Low/High & $18 / 18$ & $12 / 24$ & $0.151^{\mathrm{a}}$ \\
Marriage status: Married/unmarried & & & \\
& $32 / 4$ & $31 / 5$ & $0.722^{\mathrm{a}}$ \\
Hemiparesis: right/ left & $11 / 25$ & $19 / 17$ & $0.056^{\mathrm{a}}$ \\
\hline
\end{tabular}

${ }^{\mathrm{a}}$ chi-square

From the data above, it is known that basic characteristic of these two groups (with and without depression ) was statistically equivalent ( $p>0.05)$. The Relationship of Vascular Risk Factor with Post-stroke Depression Vascular risk factor characteristic that was connected with poststroke depression can be seen in the table below

Table 2. Vascular Risk Factor and It is Relationship with The incident of Post-Stroke Depression

\begin{tabular}{|c|c|c|c|c|c|c|}
\hline \multirow[t]{2}{*}{ Variable } & & \multicolumn{2}{|c|}{ Post-stroke Depression } & \multirow[t]{2}{*}{ Total } & \multirow[t]{2}{*}{$\mathrm{OR}$} & \multirow[t]{2}{*}{$\mathrm{p}$-value } \\
\hline & & Yes & No & & & \\
\hline \multicolumn{7}{|c|}{ Hypertension, n(\%) } \\
\hline & Yes & $25(46.3)$ & $29(53.7)$ & 54 & 0.549 & $0.276^{\mathrm{a}}$ \\
\hline & No & $11(61.1)$ & $7(38.9)$ & 18 & & \\
\hline \multicolumn{7}{|c|}{ Diabetes Mellitus, n(\%) } \\
\hline & Yes & $14(70.0)$ & $6(30.0)$ & 20 & 3.182 & $0.035^{\mathrm{a}}$ \\
\hline & No & $22(42.3)$ & $30(57.7)$ & 52 & & \\
\hline \multicolumn{7}{|c|}{ Smoking, $\mathrm{n}(\%)$} \\
\hline & Yes & $15(50.0)$ & $15(50.0)$ & 30 & 1.000 & $1.000^{\mathrm{a}}$ \\
\hline & No & $21(50.0)$ & $21(50.0)$ & 42 & & \\
\hline Total & & 37 & 37 & 74 & & \\
\hline
\end{tabular}

${ }^{\mathrm{a}} \mathrm{chi}$ square test

There is a relationship between diabetes mellitus with the incidence of post-stroke depression with a $p$-value $p=0.035$, and there is no relationship in hypertension and smoking with that incidence. 
Table 3. The Differences in mean Serum Level of Brain-Derived Neurotrophic Factor (BDNF) and Malonylaldehid (MDA) in those two group

\begin{tabular}{|c|c|c|c|}
\hline \multirow[b]{2}{*}{ Variable } & \multicolumn{2}{|l|}{ Post Ischemic Stroke } & \multirow[b]{2}{*}{$\mathrm{P}$} \\
\hline & With Depression & Without Depression & \\
\hline BDNF Level & $6442.50 \pm 1747.48$ & $7522.33 \pm 1638.45$ & $0.009^{\mathrm{a}}$ \\
\hline MDA Level & $110.06 \pm 33.27$ & $99.98 \pm 54.76$ & $0.024^{\mathrm{b}}$ \\
\hline
\end{tabular}

The Association of Brain-Derived Neurotrophic Factor (BDNF) and Malonylaldehid (MDA) Levels with Depression in Patient poststroke

The Association of Brain-Derived Neurotrophic Factor (BDNF) and Malonylaldehid (MDA) Levels with Depression in Patient poststroke is shown in table 3. The table above shows that there is a significant difference between BDNF and MDA serum level in the post-stroke group with depression and the post-stroke group without depression with a $\mathrm{p}$-value $\mathrm{p}<0.05$.

\section{Discussion}

In the basic data statistical test on both groups, the $\mathrm{p}$ value $>0.05$, which means that the basic data between case groups (stroke with depression) and control (post-stroke without depression) were statistically equivalent. This basic data includes age, gender, level of educational background, marital status and hemispheres which experienced a disturbance. Thus, between the case group and the control group which had been matched, so that the next analysis process is possible to be done. In this study, since the design of this study was case-control, the researchers did not analyze the effect of age, gender, level of educational background, marital status, and site of lesions (hemispheres) with the incidence of postischemic stroke depression.

There is a was a relationship between diabetes mellitus, in this case as a risk factor with the incidence of post-stroke depression with $p<0.05$ and diabetes mellitus patients are at risk for post-stroke depression 3.182 times greater than those who did not have diabetes mellitus.

There is a mutual correlation between diabetes and depression, which means that depression can trigger diabetes and diabetes can facilitate depression. Even though the prevalence of mental disorders is generally reported similar between the general population and diabetics patient, but the incidence of depression and anxiety is reported higher on diabetic patients [12]. Several factors associated with the incidence of depression on diabetic patients were female sex, young age, single, poor social support, low educational background, low socioeconomic status, poor glycemic control, the presence of diabetes complications and other medical comorbidities $[13,[14]$. It is known that conditions of hypoglycemia and hyperglycemia can give an adverse effect on brain function, especially in the cognitive and mood areas. In animals, it is known that diabetes affects the integrity and hippocampal neurogenesis which may also interact with other aspects of the neuroplasticity process and contribute to the symptoms of depression [15].

This study found that low serum BDNF levels were found on patients with post-stroke depression compared to the post-stroke patients without depression and this difference was statistically significant $(p=0.009)$. In general, BDNF is believed to have a beneficial effect on stroke recovery through several mechanisms: protection against acute ischemic injury, increased angiogenesis and neurogenesis, improved brain repair and increased synaptic plasticity [6]. 
The development and neuron cells sustainability in the central nervous system are regulated by many extracellular factors, one of them is the neurotrophic factor. Neurotropin has a significant effect on proliferation, migration, neurogenesis and ensuring the integrity and the function of each neuron cell. One of the neurotrophin factors is BDNF, which functions to facilitate the growth and survival of nerves cells, modulate the synapse response and is responsible for synapse plasticity processes [16]. This function can be disrupted in a variety of hypoxic conditions, where the level of disturbance correlates with the level of vascular damage in the brain. The correlation between levels of BDNF and various neurodegeneration diseases has been studied quite a lot, including its correlation to the incidence of post-stroke depression[17]. There is a decrease of mRNA BDNF expression levels in hippocampus and prefrontal cortex (PFC) on patients with depression [18]. Reduction of BDNF expression will affect structural changes such as atrophy of the hippocampus and other parts such as the amygdala, anterior cingulate cortex (ACC), orbitofrontal cortex (OFC), medial frontal cortex [19].

Study of Li et al. in 216 patients with ischemic stroke, showed that a lower serum BDNF levels at the hospital admission were aPSD independent predictors at 3 months-follow-up [20]. A meta-analysis by Noonan (2012) showed that BDNF levels were found to be much lower on patients with post-stroke depression [21].

This study also found higher MDA levels in post-stroke patients with depression compared to post-stroke patients without depression and this difference was statistically significant $(\mathrm{p}=$ $0.024)$.

The process of hypoxia that occurs in the brain due to a stroke will increase the production of free radicals. Brain tissue is a tissue that is rich of polyunsaturated fatty acids (PUFAs), because of that, a high level of free radicals will increase the production of toxic lipid peroxidation, that will mediate oxidative stress, and it will lead into death of various cell types. Some markers can measure lipid peroxidation, one of them is malondialdehyde which relatively stable compared to the other markers. Several studies have proven that free radicals are involved in the pathophysiology of depression [22].

Malondialdehyde is the final product of PUFA and arachidonic acid (AA) peroxidation. Malondialdehyde inhibits serotonin receptor ligand binding and therefore affects serotonin metabolism [22],[23]. Increased MDA concentrations can be detected on patients with depression compared to the healthy people. Intake of antidepressant is proven in reducing MDA levels [24].

This study has several weaknesses, including not analyzing the effect of vascular risk factors on BDNF and MDA levels, and also did not include other risk factors such as dyslipidemia and heart disease.

\section{Conclusion}

This study shows the association of lower level of BDNF serum and the higher level of MDA in the acute phase with the incidence of post-stroke depression. Ischemic stroke patient with diabetes mellitus has a higher risk to suffer post-stroke depression compared to a patient without diabetes mellitus. 


\section{References}

[1] Park GY, Im Sum, Lee S, and Pae CU. : The Association between Post-Stroke Depression and the Activities of Daily Living/Gait Balance in Patients with First-Onset Stroke Patients. Psychiatry Investig. Vol 13(6). pp. 659-664 (2016)

[2] Mierlo ML, Caroline M, Marcel WP, Paul L \& Johanna M. Psychological factors determine depressive symptomatology after stroke. Arch PhysMedRehabil.Vol.96(6).pp. 1064-70 (2015)

[3] Feng C, Fang M \& Liu XY. Review Article, The Neurobiological pathogenesis of poststroke depression, The Scientific World Journal, Vol 2014.Article ID: 521349. (2014)

[4] Teixeira AL, Barbosa IG, Diniz BS \& Kummer A. Circulating levels of brain-derived neurotrophic factor: correlation with mood, cognition and motor function. Biomarkers Med. Vol. 4, No. 6. Pp. 871-887 (2010)

[5] Marosi K, Mattson MP. BDNF mediates adaptive brain and body responses to energetic challenges. Trends EndocrinolMetab. pp. 1-10 (2013)

[6] Zhang ZH, Wu LN, Song JG \& Li WQ. Correlations between cognitive impairment and brain-derived neurotrophic factor expression in the hippocampus of post-stroke depression rats. Molecular medicine reports.Vol 6, pp. 889-893 (2012)

[7] Kontush A. Lipid peroxidation and Alzheimer's Disease: Key Role of Amyloid - $\beta$. Lipides, OCL VOL. $13 \mathrm{~N}^{\circ} 1$ JANVIER-FE'VRIER. pp 46-53 (2006)

[8] Leonard B and Maes M. Mechanistic explanations how cellmediated immune activation, inflammation and oxidative and nitrosative stress pathways and their sequels and concomitants play a role in the pathophysiology of unipolar depression. Neurosci Biobehav Rev. vol. 36. no. 2. pp. $764-785$ (2012.)

[9] Rybka JK, Kedziora KP, BanasLI, Majsterek LA, Carvalho A, Cattaneo CA, Kedziora. Interplay between the pro-oxidant and antioxidant systems and proinflammatory cytokine levels, in relation to iron metabolism and the erythron in depression. Free Radic Biol Med. Vol 63. pp. 187-194 (2013)

[10] Stefanescu CA, Ciobica. The relevance of oxidative stress status in first episode and recurrent depression. J Affect Disord. vol 143(1-3). pp. 34-38 (2012)

[11] Michalakeas CA,. Parissis JT, Douzenis A, Nikolaou M, Varounis C, Andreadou I, Antonellos $\mathrm{N}$, et al. Effects of sertraline on circulating markers of oxidative stress in depressed patients with chronic heart failure: a pilot study. J Card Fail. Vol 17(9). pp. 748-754 (2011)

[12] Kruse J, Schmitz N, Thefeld W. On the association between diabetes and mental disorders in a community sample. Results from the German National Health Interview and Examination Survey. Diabetes Care. Vol 26. pp. 1841-1846 (2003)

[13] Engum A, Mykletun A, Midthjell K, Holen A, Dahl AA. Depression and Diabetes. A large population-based study of sociodemographic, lifestyle and clinical factors associated with depression in type 1 and type 2 diabetes. Diabetes Care.vol 28. pp. 1904-1909 (2005)

[14] Katon W, Fan MY, Unützer J, Taylor J, Pincus H, Schoenbaum M. Depression and diabetes: a potentially lethal combination. J Gen Intern Med. Vol 23. pp. 1571-1578 (2008)

[15] Ho N, Sommers MS, Lucki I. Effects of diabetes on hippocampal neurogenesis: links to cognition and depression. Neurosci Biobehav Rev. vol 37. pp. 1346-62 (2013)

[16] Huang EJ, Reichardt LF: Neurotrophins: roles in neuronal development and function. Annu Rev Neurosc. vol 24. pp. 677-736 (2001)

[17] Anetta Lasek-Bal, Halina Jędrzejowska-Szypułka, Jagoda Różycka, Wiesław Bal, Michał Holecki, Jan Duława, Joanna Lewin-Kowalik. Low Concentration of BDNF in the Acute Phase of Ischemic Stroke as a Factor in Poor Prognosis in Terms of Functional Status of Patients. Med Sci Monit. vol 21.pp. 3900-3905 (2015)

[18] Autry AE, Monteggia LM. Brain derived neurotrophic factor and neuropsychiatric dissorder. Pharmacology Rev.vol 64.pp. 238-258 (2012)

[19] Sakata K. Brain-derived neurotrophic factor and major depression. Neurobiology Depression. CRC Press. pp. 391-419 (2012)

[20] Li J, Zhao YD, Zeng JW, Chen XY \& Wang RD. Serum brain-derived neurotrophic factor levels in post-stroke depression. J Affect Disord. Vol.168. pp. 373-379 (2014) 
[21] Noonan K, Carey LM \& Crewther SG. Meta-analyses indicate associations between neuroendocrine activation, deactivation in neurotrophic and neuroimaging markers in depression after stroke. J Stroke CerebrovascDis. Vol. 09, No.008. pp: 1-12 (2012)

[22] Leonard B and Maes M. Mechanistic explanations how cellmediated immune activation, inflammation and oxidative and nitrosative stress pathways and their sequels and concomitants play a role in the pathophysiology of unipolar depression. Neurosci Biobehav Rev. vol. 36, no. 2. pp. $764-785$ (2012)

[23] Scapagnini G, Davinelli S, Drago F, De Lorenzo A, and Oriani G. Antioxidants as antidepressants: fact or fiction?. CNS Drugs. vol. 26. no. 6. pp. 477-490 (2012)

[24] Gałecki P, Szemraj J, Bie'nkiewicz M, Florkowski A, and Gałecka E. Lipid peroxidation and antioxidant protection in patients during acute depressive episodes and in remission after fluoxetine treatment. Pharmacol Rep.vol. 61. no. 3. pp. 436-447(2009) 